\title{
Scalar flux profile relationships over the open ocean
}

\author{
J. B. Edson, C. J. Zappa, ${ }^{1}$ J. A. Ware, and W. R. McGillis ${ }^{2}$ \\ Woods Hole Oceanographic Institution, Woods Hole, Massachusetts, USA \\ J. E. Hare $^{3}$ \\ Environmental Technologies Laboratory, National Oceanic and Atmospheric Administration, Boulder, Colorado, USA \\ Received 15 May 2003; revised 5 March 2004; accepted 14 May 2004; published 14 August 2004.
}

[1] The most commonly used flux-profile relationships are based on Monin-Obukhov (MO) similarity theory. These flux-profile relationships are required in indirect methods such as the bulk aerodynamic, profile, and inertial dissipation methods to estimate the fluxes over the ocean. These relationships are almost exclusively derived from previous field experiments conducted over land. However, the use of overland measurements to infer surface fluxes over the ocean remains questionable, particularly close to the ocean surface where wave-induced forcing can affect the flow. This study investigates the flux profile relationships over the open ocean using measurements made during the 2000 Fluxes, Air-Sea Interaction, and Remote Sensing (FAIRS) and 2001 GasEx experiments. These experiments provide direct measurement of the atmospheric fluxes along with profiles of water vapor and temperature. The specific humidity data are used to determine parameterizations of the dimensionless gradients using functional forms of two commonly used relationships. The best fit to the Businger-Dyer relationship [Businger, 1988] is found using an empirical constant of $a_{q}=13.4 \pm 1.7$. The best fit to a formulation that has the correct form in the limit of local free convection [e.g., Wyngaard, 1973] is found using $a_{q}=29.8 \pm 4.6$. These values are in good agreement with the consensus values from previous overland experiments and the Coupled Ocean-Atmosphere Response Experiment (COARE) 3.0 bulk algorithm [Fairall et al., 2003]; e.g., the COARE algorithm uses empirical constants of 15 and 34.2 for the Businger-Dyer and convective forms, respectively. Although the flux measurements were made at a single elevation and local similarity scaling is applied, the good agreement implies that MO similarity is valid within the marine atmospheric surface layer above the wave boundary layer. INDEX TERMS: 0312 Atmospheric Composition and Structure: Air/sea constituent fluxes (3339, 4504); 1655 Global Change: Water cycles (1836); 3307 Meteorology and Atmospheric Dynamics: Boundary layer processes; 4247 Oceanography: General: Marine meteorology; KEYWORDS: air-sea fluxes, flux profile relationships, marine boundary layer

Citation: Edson, J. B., C. J. Zappa, J. A. Ware, W. R. McGillis, and J. E. Hare (2004), Scalar flux profile relationships over the open ocean, J. Geophys. Res., 109, C08S09, doi:10.1029/2003JC001960.

\section{Introduction}

[2] Over the ocean, direct measurement of turbulent fluxes is often complicated by platform motion, flow distortion, and the effects of sea spray. Because of this, marine meteorologists and oceanographers have long relied on flux profile relationships that relate the turbulence fluxes of momentum, heat, and moisture (or mass) to their respective profiles of velocity, temperature, and water vapor (or

\footnotetext{
${ }^{1}$ Now at Ocean and Climate Physics Division, Lamont-Doherty Earth Observatory, Columbia University, Palisades, New York, USA.

${ }^{2}$ Now at Geochemistry Division, Lamont-Doherty Earth Observatory, Columbia University, Palisades, New York, USA.

${ }^{3}$ Also at Cooperative Institute for Research in Environmental Sciences, University of Colorado, Boulder, Colorado, USA.

Copyright 2004 by the American Geophysical Union. 0148-0227/04/2003JC001960
}

other gases). These flux profile relationships are required in indirect methods such as the bulk aerodynamic, profile, and inertial dissipation methods that estimate the fluxes from mean, profile, and high-frequency spectral measurements, respectively. The flux profile or gradient flux relationships are also used extensively in numerical models to provide lower boundary conditions and to "close" the model by approximating higher-order terms from low-order variables. The most commonly used flux-profile relationships are based on Monin-Obukhov (MO) similarity theory. MO similarity theory predicts that the nondimensional gradient of velocity, temperature, and humidity are universal functions of atmospheric stability

$$
\frac{\kappa z}{x_{*}} \frac{\partial X}{\partial z}=\phi_{x}\left(\frac{z}{L}\right)
$$


where $x=u, \theta, q$ denotes velocity, potential temperature, and specific humidity, respectively; $x_{*}$ is the scaling parameter for the variable $x ; \kappa$ is von Karman's constant $z$; is the height above the surface; $X$ represents the mean of the variable $x$; and $z / L$ is a stability parameter where $L$ is the MO length. The stability parameter $-z / L$ represents the ratio of buoyant production (convection) to mechanical production (shear) in a constant flux layer. As such, it is closely related to the flux Richardson number, which is more commonly used in investigation of the oceanic mixed layer.

[3] These flux-profile relationships have been investigated during overland experiments including the tower studies by Dyer and Hicks [1970] and Carl et al. [1973], the landmark Kansas and International Turbulence Comparision (ITCE) experiments [e.g., Businger et al., 1971; Dyer and Bradley, 1982], and more recently in the experiment described by Högström [1988], Frenzen and Vogel [1992, 2001], Oncley et al. [1996], and Poulos et al. [2002]. These and other experiments have generated a number of similar semiempirical functions, with the most commonly used forms known as the Businger-Dyer formulae [Businger, 1988].

[4] While the majority of the investigations were conducted over land, several notable exceptions have been conducted over water. Badgley and Paulson [1972] and Paulson et al. [1972a] describe measurements of wind, temperature, and humidity profiles over the Arabian Sea during the 1964 International Indian Ocean Expedition. They report results that are consistent with Businger-Dyer formulae. However, they did not directly measure the fluxes, so they could not directly investigate the flux-profile relationships. Miyake et al. [1970] measured turbulent fluxes and profiles from two masts located in a fetch limited location over the sea. They found the profile and direct covariance estimates to agree well within the experimental error using Businger-Dyer formulae. However, they did not attempt to refine the flux-profile relationships, presumably because of the limited range of stability. Pond et al. [1971] and Paulson et al. [1972b] measured velocity, temperature, and humidity profiles and their associated fluxes during Barbados Oceanographic and Meteorological Experiment (BOMEX) aboard the R/P FLIP. However, uncertainty in their velocity profiles caused by flow distortion around FLIP as well as salt contamination of the temperature profiles and flux estimates also limited their analyses to comparisons of profile and direct covariance flux estimates using Businger-Dyer formulae.

[5] As a result, the semiempirical formulae developed over land remain widely used in indirect methods such as the bulk aerodynamic and inertial dissipation methods to estimate the turbulent fluxes over the ocean. However, the use of overland measurements to infer surface fluxes over the ocean remains questionable, particularly close to the ocean surface where wave-induced forcing can affect the flow. For example, recent investigations of the dimensionless shear over the ocean by Miller et al. [1997], Vickers and Mahrt [1999], and Smedman et al. [1999] report waveinduced effects that can cause substantial departure from MO similarity predictions. Therefore the universality of these relationships to all surface layers is a current topic of intense debate.
[6] This paper investigates scalar flux profiles over the open ocean using data from two field experiments. The atmospheric stability during these experiments was predominantly unstable and the analysis is limited to an investigation of the dimensionless profile functions under these conditions. A brief description of the underlying principles used in MO similarity theory followed by a more detailed discussion of the semiempirical functions is provided in section 2. The experimental setups used during these two experiments are described in section 3 . Section 4 presents the results from the experiments and compares them with previous investigations over land and water.

\section{Monin-Obukhov Similarity Theory}

[7] Monin-Obukhov similarity theory provides a powerful statistical tool for studies of atmospheric turbulence in the surface layer. At its foundation is the assumption that flows with similar ratios of convective to mechanical generation of turbulence at a given height (i.e., similar Richardson numbers) are expected to have similar turbulent structures. Specifically, MO similarity theory states that the structure of turbulence in a constant flux layer is determined by the height above the surface, $z$; the buoyancy parameter, $g / \Theta_{v}$, where is gravity and $\Theta_{v}$ is the mean virtual potential temperature; the friction velocity, $u_{*}$, and the surface buoyancy flux, $\overline{w \theta_{v s}}$, where and $\theta_{v}$ are the fluctuating vertical velocity and virtual potential temperature, respectively, and the subscript denotes the surface value. The friction velocity is derived from the surface stress

$$
u_{*}=\left[\frac{\tau_{s}}{\rho_{a}}\right]^{1 / 2}=\left|F_{u}(0)\right|^{1 / 2}
$$

while the buoyancy flux can be broken down as

$$
\overline{w \theta_{v s}}=\frac{Q_{h}}{\rho_{a} c_{p}}+0.61 T_{s} \frac{Q_{e}}{\rho_{a} L_{e}}=F_{\theta}(0)+0.61 T_{s} F_{q}(0)
$$

where $\tau_{s}$ is the surface stress vector, $Q_{h}$ is the surface value of the sensible heat flux, $Q_{e}$ is the surface value of the latent heat flux, $T_{s}$ is the surface temperature, $c_{p}$ is the specific heat at constant pressure, $L_{e}$ is the latent heat of vaporization of water, and, $F_{u}, F_{\theta}$ and $F_{q}$ are the kinematic forms [e.g., see Stull, 1988] of the momentum, sensible heat, and latent heat fluxes, respectively. These quantities define the additional scaling parameters as

$$
x_{*} \equiv-\frac{F_{x}(0)}{u_{*}}
$$

as well as the MO length

$$
L=-\frac{\Theta_{v}}{g \kappa} \frac{u_{*}^{3}}{\overline{w \theta_{v_{s}}}}=\frac{\Theta_{v}}{g \kappa} \frac{u_{*}^{2}}{\left(\theta_{*}+0.61 T_{s} q_{*}\right)}
$$

The similarity hypothesis then states that various turbulent statistics, when normalized by these scaling parameters, are universal functions of $z / L$. 
[8] These functions are commonly used to provide indirect estimates of the surface fluxes over the ocean. For example, equations (1) and (4) can be combined to provide parameterizations for the fluxes given by

$$
F_{x}=-\frac{u_{*} \kappa z}{\phi_{x}(\zeta)} \frac{\partial X}{\partial z}=K_{x} \frac{\partial X}{\partial z}
$$

where, $\zeta=z / L$, and $K_{x}$ are the eddy diffusivities as defined using MO similarity. This parameterization is the basis for the gradient flux method. Note that the eddy diffusivities for momentum and heat, denoted by $K_{u}$ and $K_{\theta}$, are more commonly denoted by $K_{m}$ and $K_{h}$. The notation used in equation (6) avoids mixing subscripts and is therefore employed for simplicity.

[9] These scaling laws are expected to hold and the derived parameterizations are expected to be universal as long as the assumptions that govern the MO similarity laws are valid, i.e., a combination of mechanical and thermal forcing drive the turbulent exchange, the scaling variables are independent of height in the surface layer, and the turbulence statistics are stationary and horizontally homogeneous. The constant flux layer constraint is generally assumed to be valid in the lowest $10 \%$ of the unstable atmospheric boundary layer.

[10] In highly stratified boundary layers where large flux divergences are observed even close to the surface, the constant flux assumption is often relaxed resulting in an extension of MO similarity known as local similarity theory [cf. Mahrt, 1998]. Local similarity uses the local value of the fluxes to define scaling parameters that may vary with height. An accurate implementation of this approach requires normalization of the gradients using local values of the gradient and scaling parameters. This is also the most accurate application of MO similarity theory when only one level of flux estimates is available, i.e., the local unadjusted values of the fluxes are used to scale the local gradients computed at the level of the flux estimates. This describes the experimental setup used in the investigations, i.e., the fluxes are computed at a single elevation. Therefore this paper uses the local similarity approach to investigate functional forms of the dimensionless water vapor profiles given by equation (1).

[11] Finally, these assumptions can also become invalid in the lowest $10 \%$ of the marine boundary layer when wave induced flow plays an important role in turbulent exchange. This near surface region of the marine boundary layer is referred to as the wave boundary layer (WBL). At present, there is no consensus definition for the height of the WBL. For example, many numerical modelers assume that the influence of the waves is limited to the region where $z / \sigma_{H} \ll 1$, where $\sigma_{H}$ is the significant wave height of the dominant waves [e.g., Janssen, 1989; Makin et al., 1995]. However, recent field campaigns have shown that some turbulent statistics, e.g., the pressure transport term in the kinetic energy budget equation [Hare et al., 1997; Edson and Fairall, 1998; Hristov et al., 2003], are influenced by waves up to heights where $k_{p} z \approx 2$, where is the peak wave number of the dominant waves. The latter findings suggest a substantially thicker WBL for some characteristics of the flow.
[12] Most investigations assume that the transfer of heat and mass is less affected by ocean waves than the transfer of momentum and kinetic energy. The physical argument behind this assumption is that the momentum flux can be carried by both tangential stress and the normal stress, or form drag, created by interaction between the pressure and wave fields. The form drag and energy input to the waves appear as source/sink term in the momentum and kinetic energy budgets, respectively [e.g., Edson and Fairall, 1998; Janssen, 1999]. However, the equivalent of a normal stress does not exist in the heat or scalar variance budgets due to the absence of pressure terms. This translates to the absence of a wave-induced heat or mass flux over waves [Makin et al., 1995].

[13] This does not mean that the effect of waves on heat and mass transfer is necessarily negligible since the eddy diffusivities in equation (6) are expected to be modulated by waves [e.g., Makin and Mastenbroek, 1996]. As a result, the turbulent flux of heat and mass is also expected to be modulated by waves. Additionally, the presence of evaporating sea-spray is expected to influence the transfer of heat and mass at high winds [e.g., Fairall et al., 1994; Makin, 1998; Andreas and Emanuel, 2001]. However, there is considerable debate about the type of conditions required to observe a significant impact on the fluxes.

\subsection{Functional Forms for Unstable Atmospheric Boundary Layers}

[14] Forty years of experimental dimensionless profile investigations have lead to a myriad of parameterizations and even more investigations to address the universality of these functions. However, it has become common practice to parameterize the dimensionless profiles in unstable conditions using

$$
\phi_{x}(\zeta)=\gamma_{x}\left(1-a_{x} \zeta\right)^{-N_{x}}
$$

where $a_{x}$ and $N_{x}$ are numerical constants and $\gamma_{x}$ is the value of the dimensionless profile under neutral conditions. The values of the dimensionless temperature and humidity profiles under neutral conditions are closely related to the turbulent Prandtl and Schmidt numbers, respectively. For example, the turbulent Schmidt number, $S c$, is defined as the ratio of the eddy diffusivity for momentum, $K_{u}$, over the eddy diffusivity for mass, $K_{q}$. MO similarity predicts that the ratio of the eddy diffusivities is related to the dimensionless functions as

$$
S c=\frac{K_{u}}{K_{q}}=\frac{\phi_{q}(\zeta)}{\phi_{u}(\zeta)}
$$

Therefore the neutral values of the dimensionless function are related by

$$
\frac{\gamma_{x}}{\gamma_{u}}=\frac{K_{u}(\zeta=0)}{K_{x}(\zeta=0)}=\frac{\phi_{x}(0)}{\phi_{u}(0)}
$$

such that by definition the neutral value of the dimensionless shear, $\gamma_{u}$, is equal to 1 . This condition sets the value of the von Karman's constant $\kappa$. This relationship also equates 
Table 1. Numerical Constants for the Businger-Dyer Formulae From Previous Field Experiments

\begin{tabular}{|c|c|c|c|c|c|c|c|c|}
\hline \multirow[b]{2}{*}{ Investigation } & \multicolumn{4}{|c|}{ Experimental } & \multicolumn{2}{|c|}{$\begin{array}{c}\text { Adjusted } \\
\text { According to } \\
\text { Yaglom }[1977]\end{array}$} & \multicolumn{2}{|c|}{$\begin{array}{c}\text { Adjusted } \\
\text { According to } \\
\text { Högström [1988] }\end{array}$} \\
\hline & $\kappa$ & $\gamma_{y}$ & $a_{u}$ & $a_{y}$ & $a_{u}$ & $a_{y}$ & $a_{u}$ & $a_{y}$ \\
\hline Dyer and Hicks [1970] & 0.41 & 1.0 & 16 & 16 & 16.4 & 16.4 & 16 & 16 \\
\hline Businger et al. [1971] & 0.35 & 0.74 & 15 & 9 & 13.1 & 7.9 & 20.3 & 12.2 \\
\hline Dyer and Bradley [1982] & 0.40 & 1.0 & 28 & 14 & 28 & 14 & 28 & 14 \\
\hline Oncley et al. [1996] & 0.37 & 0.86 & 15 & 9 & 13.7 & 8.2 & 17.4 & 10.5 \\
\hline Frenzen and Vogel [2001] & 0.38 & - & 21 & - & 20.1 & - & 21 & - \\
\hline Average of Values & 0.38 & 0.9 & 21 & 12 & 18.3 & 11.6 & 20.5 & 13.2 \\
\hline
\end{tabular}

the neutral value of the turbulent Schmidt number, $S c_{0}$, with the neutral value of the dimensionless humidity profile, $\gamma_{q}$.

[15] There is considerable consensus in the meteorological community on the value of the exponential constant in equation (7). In unstable conditions over the range $-2<$ $z / L<0$, a number of field experiments [e.g., Dyer and Hicks, 1970; Businger et al., 1971; Dyer and Bradley, 1982] have lead to the empirical relationships

$$
\frac{K_{u}}{K_{y}}=\gamma_{y} \phi_{u}(\zeta)
$$

and

$$
\phi_{u}(\zeta)=\left(1-a_{u} \zeta\right)^{1 / 4}
$$

where $y=\theta, q$ and $a_{u}$ is the numerical constant for the velocity profile. The combination of equations (8), (10), and (11) results in a value of $1 / 2$ for $N_{y}$ in the parameterization of both $\phi_{\theta}$ and $\phi_{q}$. The scalar parameterizations using $N_{y}=$ $1 / 2$ have also been shown to give good agreement with potential temperature observations over the stability range $-2<z / L<0$ by these investigators. This set of functions, i.e., with $N_{u}=1 / 4$ and $N_{y}=1 / 2$, are known as BusingerDyer formulae [Businger, 1988].

[16] The dimensionless profiles for all variables are expected to become proportional to $(-\zeta)^{1 / 3}$ in the limit of free convection [Gurvich, 1965; Wyngaard, 1973]. Therefore several formulae with $N_{x}=1 / 3$ have been proposed [e.g., Carl et al., 1973; Frenzen and Vogel, 2001]. While these formulae often give good agreement with data in very unstable conditions, they are inferior to the Businger-Dyer formulae near neutral conditions. The KEYPS equation [e.g., Panofsky et al., 1960] provided a formula that incorporated the observed near neutral conditions with the expected behavior in convective limit. However, this equation is difficult to use in practice to estimate the fluxes from indirect methods. A less elegant but more easily implemented approach is used in the Tropical Ocean-Global Atmosphere (TOGA)-Coupled Ocean-Atmosphere Response Experiment (COARE) bulk algorithm [Fairall et al., 1996]. The algorithm smoothly blends the Businger-Dyer and convective forms to provide a parameterization with the expected behavior in both nearneutral and convective conditions [Grachev et al., 2000].

[17] On the other hand, there remains considerable disagreement on the values for $\kappa, \gamma_{y}, a_{u}$ and $a_{y}$ as shown in Table 1. Recent studies by Oncley et al. [1996], Frenzen and Vogel [2001], and Andreas et al. [2002] have suggested that the von Karman's constant is actually a variable that depends on the roughness Reynolds number, $R e_{o}=z_{o} u_{*} / \nu$, where $z_{o}$ is the roughness length and is the kinematic viscosity. If true, one would expect to find different values of the numerical constants at different sites due to the inclusion of the von Karman's constant in the definition of $L$ [cf. Yaglom, 1977]. Yaglom [1977] and Högström [1988] developed approaches to modify functions from different experiments such that they used the same values for $\kappa$ and $\gamma_{y}$. As shown in Table 1, Högström's [1988] approach reduces the differences between the various formulae after adjustments to the coefficients that assume $\gamma_{y}=1$ and $\kappa=0.4$. The inclusion of several field programs since his study provide consensus values of 13.2 and 20.5 for $a_{y}$ and $a_{u}$, respectively.

\section{Experiment Setup}

[18] Direct measurements of the atmospheric fluxes along with profiles of water vapor and temperature were made during the 2000 Fluxes, Air-Sea Interaction, and Remote Sensing (FAIRS) and 2001 GasEx experiments. The FAIRS experiment took place from 15 September to 15 October 2000 aboard the R/P FLIP. The data used in the present analysis were taken during the first leg of the cruise from 23 to 30 September (yeardays 267 to 274). The FLIP was allowed to drift during this experiment and was located about $200 \mathrm{~km}$ west of the central California coast centered around $34^{\circ} \mathrm{N}, 124^{\circ} \mathrm{W}$. The GasEx-01 experiment took place in the equatorial Pacific aboard the National Oceanic and Atmospheric Administration (NOAA) R/V Ronald H. Brown. The data used in this investigation was collected from 14 to 28 February 2001 (yeardays 45 to 60). The R/V Brown operated just south of the equator at $3^{\circ} \mathrm{S}$ between the longitudes $122^{\circ} \mathrm{W}$ to $150^{\circ} \mathrm{W}$.

\subsection{Direct Covariance Fluxes}

[19] A flux package was deployed during the FAIRS experiment on a mast that placed the sensors $3 \mathrm{~m}$ above the port boom as shown in Figure 1. The mast was located roughly $15 \mathrm{~m}$ from FLIP's superstructure and $13.3 \mathrm{~m}$ above the ocean surface. The package included a three-axis sonic anemometer/thermometer (Solent R2), an open path infrared hygrometer $/ \mathrm{CO}_{2}$ sensor (Licor 7500), a three-axis accelerometer/angular rate sensor (Systron Donner MotionPak), magnetic compass (Precision Navigation), and a relative humidity/temperature sensor (Vaisala HMP45). The velocity measurements were motion-corrected using the approach given by Edson et al. [1998]. Turbulent fluxes of momentum, heat, and moisture were computed over 15-min intervals. Time series of the turbulent fluxes used in this investigation are shown in Figure 2. 


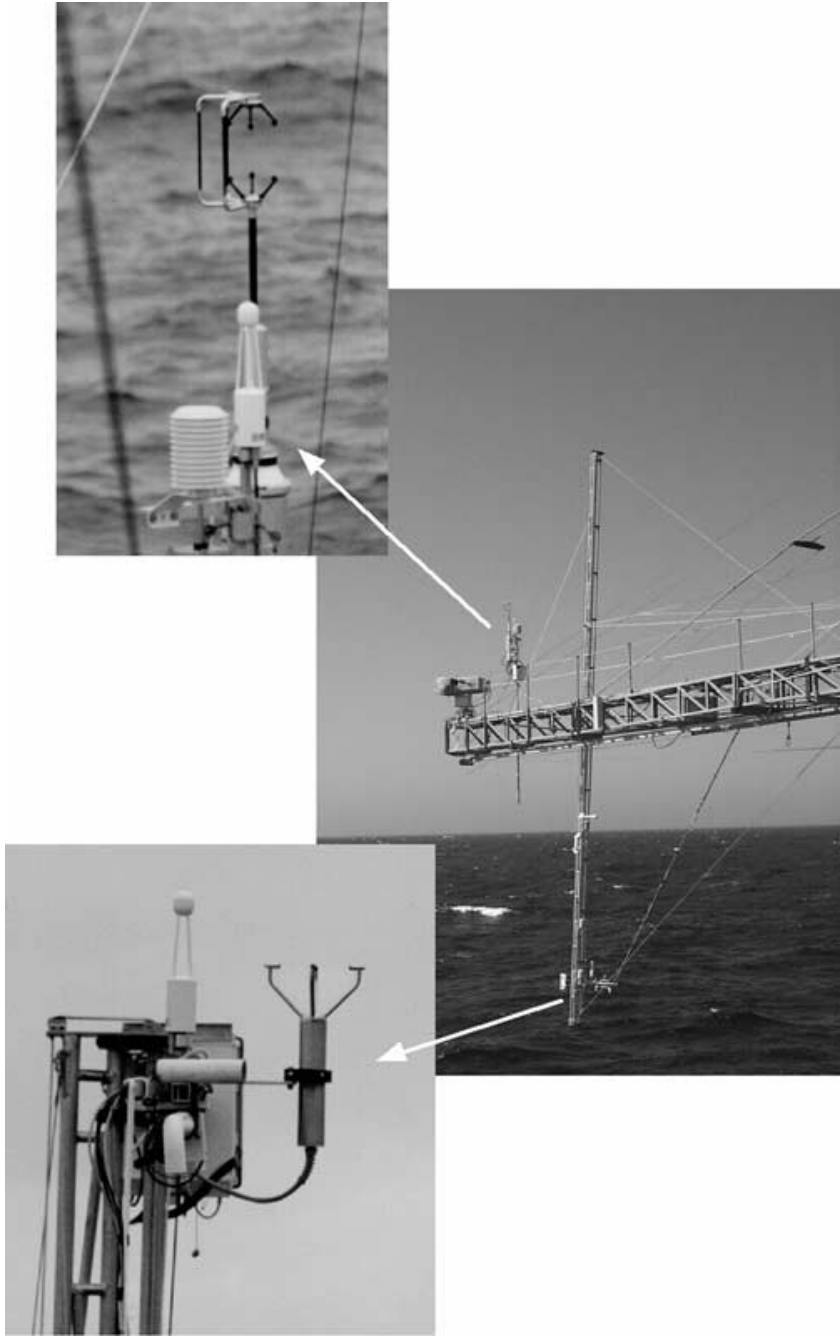

Figure 1. Composite images of the experimental setup used during the Fluxes, Air-Sea Interaction, and Remote Sensing (FAIRS) experiment. The upper left image shows the three-axis sonic anemometer-thermometer and infrared hygrometer used to compute the fluxes. The central image shows the profiling mast as deployed on the port boom of the R/P FLIP. The lower left image shows the package that traveled up and down the profiler during the experiment.

[20] During the GasEx-01 experiment, turbulent flux measurements were made from the end of a boom that placed the sensors $10 \mathrm{~m}$ upwind of the bow as shown in Figure 3. Turbulent fluxes of momentum, heat, and water vapor were made by two flux packages: one was identical to the FAIR's package, while the other used a Solent R3 anemometer and Crossbow DMU motion-sensing package. Time series of the fluxes from these packages are shown in Figure 4. The fluxes from the Solent R2 package were used for relative wind directions to starboard of bow-on flow, while the fluxes from the Solent R3 package were used for relative wind directions to port of bow-on flow.

\subsection{Profile Measurements}

[21] During FAIRS, profile measurements of velocity, temperature, and humidity were computed using a suite of sensors moving up and down a 12-m mast. The mast was suspended off of FLIP's port boom, roughly $15 \mathrm{~m}$ from the superstructure as shown in Figure 1. The top and bottom of the mast were located 4.5 and $16.5 \mathrm{~m}$ above the mean sea surface, respectively. The sensor suite included an aspirated relative humidity/temperature sensor (Vaisala HMP233), an open path infrared hygrometer/ $\mathrm{CO}_{2}$ sensor (Licor 7500 Infrared Gas Analyzer (IRGA)), an aspirated thermocouple (Campbell Scientific Model ASPTC), and a two-axis sonic anemometer (Solent Wind Observer). Additionally, a closed path infrared hygrometer/ $\mathrm{CO}_{2}$ sensor (Licor 6262 IRGA) made measurements by sampling air through a tube attached to the moving sensor package. A fixed suite of sensors that included aspirated relative humidity and temperature sensors was deployed $13.3 \mathrm{~m}$ above the sea surface on the $12-\mathrm{m}$ mast. These sensors were used to remove naturally occurring variability during the profiling periods as described below.

[22] During GasEx-01, a mast at the end of the $10-\mathrm{m}$ boom supported a profiling system that moved a suite of sensors between 3 and $12 \mathrm{~m}$ above the mean sea level as shown in Figure 5. The sensor suite did not include an anemometer but was otherwise identical to the package used in FAIRS. As in FAIRS, the moving sensors were referenced against a fixed suite of sensors located at $9 \mathrm{~m}$. A single profile took approximately 1 hour to complete. Naturally occurring temporal variability over this sampling period often generated larger differences than the differences due to the gradient as shown in Figure 6. Most of the naturally occurring temporal variability in water vapor was removed by referencing each measurement at a given height versus a fixed sensor. The difference between the fixed and traveling sensors is shown in the lower time series in Figure 6 . The results indicate that this approach removes most of the temporal variability such that the gradient in the water vapor profile is clearly visible. The main advantage of
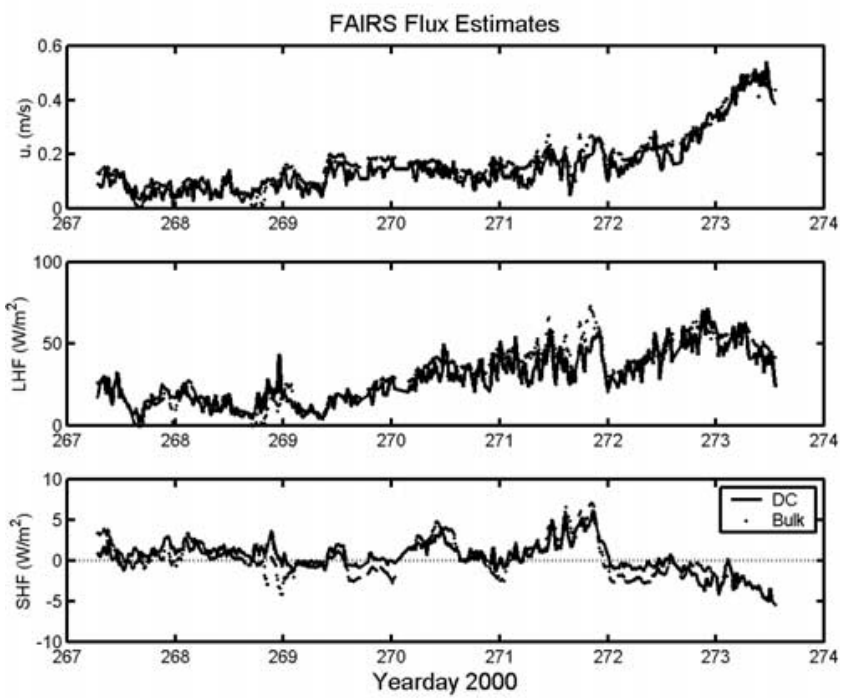

Figure 2. Time series of the fluxes derived from the direct covariance and bulk aerodynamic methods during FAIRS. The upper panel is the friction velocity derived from the stress estimates and the middle and lower panels are the latent heat and sensible heat fluxes, respectively. 


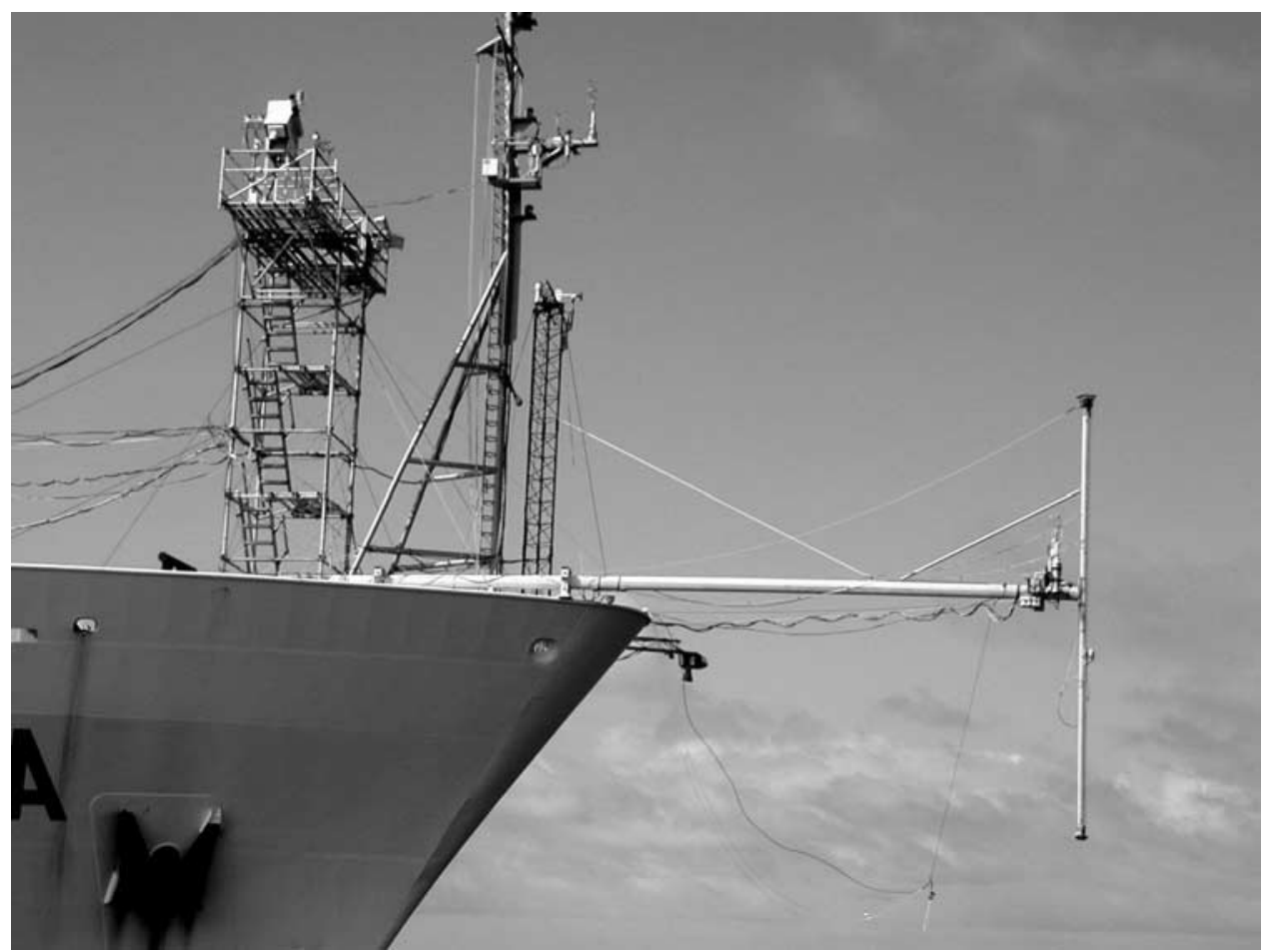

Figure 3. The experiment setup used during the GasEx experiment aboard the R/V Ronald Brown. The mast extended $10 \mathrm{~m}$ forward of the bow at its closest point. The figure also shows the flux package deployed by National Oceanic and Atmospheric Administration Environmental Technology Laboratory on the forward jackstaff and the infrared imagery system deployed by University of Washington Applied Physics Laboratory on the scaffolding.

this approach over fixed sensors is that it reduces the calibration errors that arise between sensors due to drift and degradation at sea.

[23] This approach assumes that the temporal variability is uniform over the measurement heights. To test this assumption, the ratio of the standard deviation of the profile to reference specific humidity measurements over the course of the two experiments is shown in Figure 7. The FAIRS results are consistent with the observation that the Licor measurements were substantially noisier than the Vaisala measurements due to difficulties with securing the sampling tubes, particularly at the lowest levels. The GasEx-01 measurements show less variability in both profiles. However, both experiments indicate that the uncertainty due to this assumption increases near the surface. We estimate the uncertainty due to this assumption is approximately $5-10 \%$.

[24] The Vaisala relative humidity and temperature sensors were calibrated before the cruises. A secondary in situ calibration of the profiling sensors (including the closed path IRGAs) against the reference sensors was conducted during postanalysis. The secondary calibration amounted to a small correction to the Vaisala sensors and removal of a bias in the IRGAs. The vertical profiles were computed from a least squares fit to

$$
\left[Q\left(z, t_{i}\right)-Q_{r}\left(t_{i}\right)\right]+\frac{1}{N} \sum_{i=1}^{N} Q_{r}\left(t_{i}\right)=b_{0}+b_{1} \ln (z)+b_{2}[\ln (z)]^{2}
$$

where $Q_{r}$ and $Q$ are the average values at the reference and profile height $z$, respectively, over the sampling interval at each height $t_{i}$, and $N$ is the total number of sampling intervals. Therefore the last term on the left-hand side is the mean value of the humidity over the profiling period. The gradient is then determined by differentiating the right-hand side of equation (12) to find $d Q / d \ln (z)$. Individual profiles from the Licor and Vaisala sensors are used to compute two
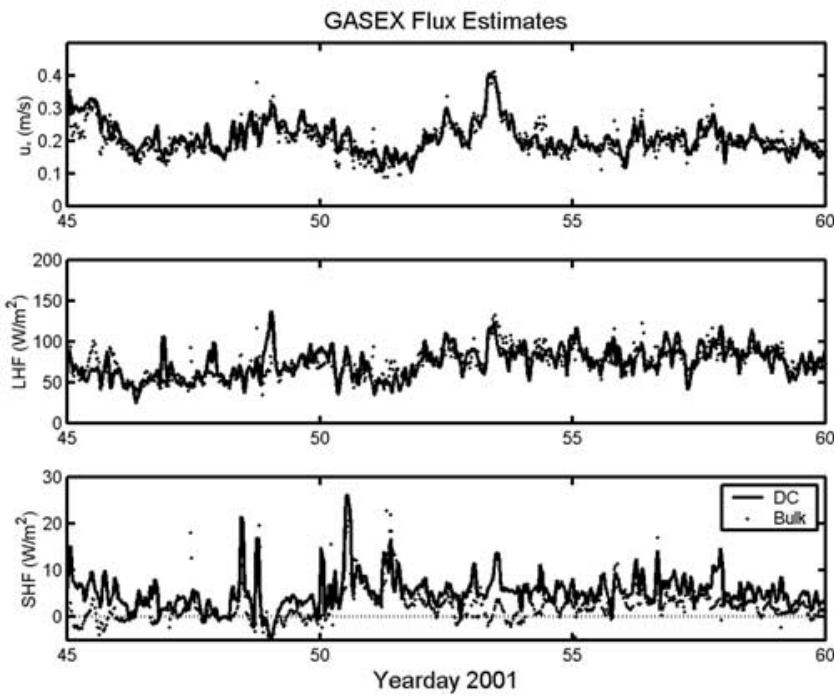

Figure 4. As in Figure 2 for the GasEx experiment. 


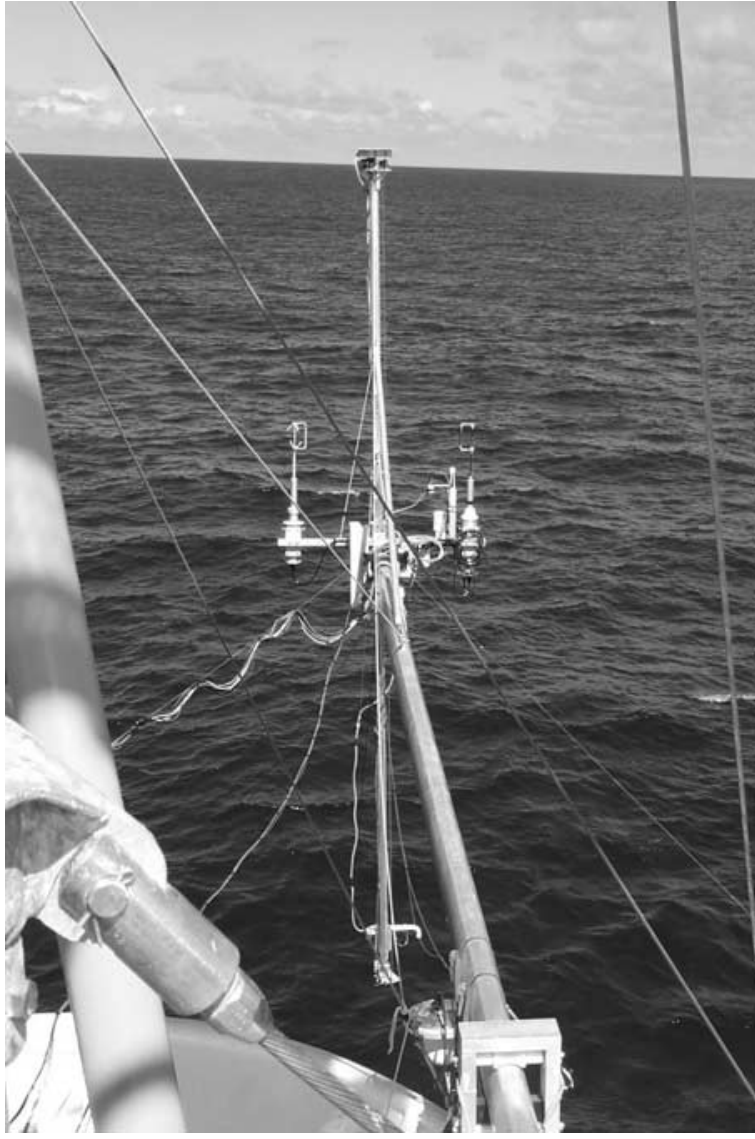

Figure 5. A photo of the profiling mast and flux sensors taken from the bow of the R/V Brown. The profiling sensors can be seen at their lowest position on the mast.

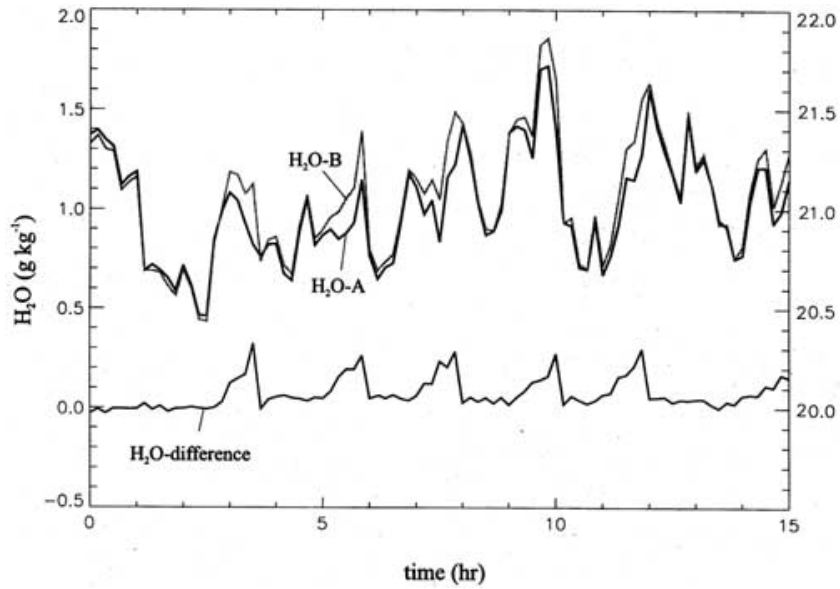

Figure 6. Time series of the specific humidity measurements taken from the profiling system. The upper time series shows the fixed reference package (thick line) and profiling package (thin line). The lower time series shows the difference between the profiling and fixed reference package.
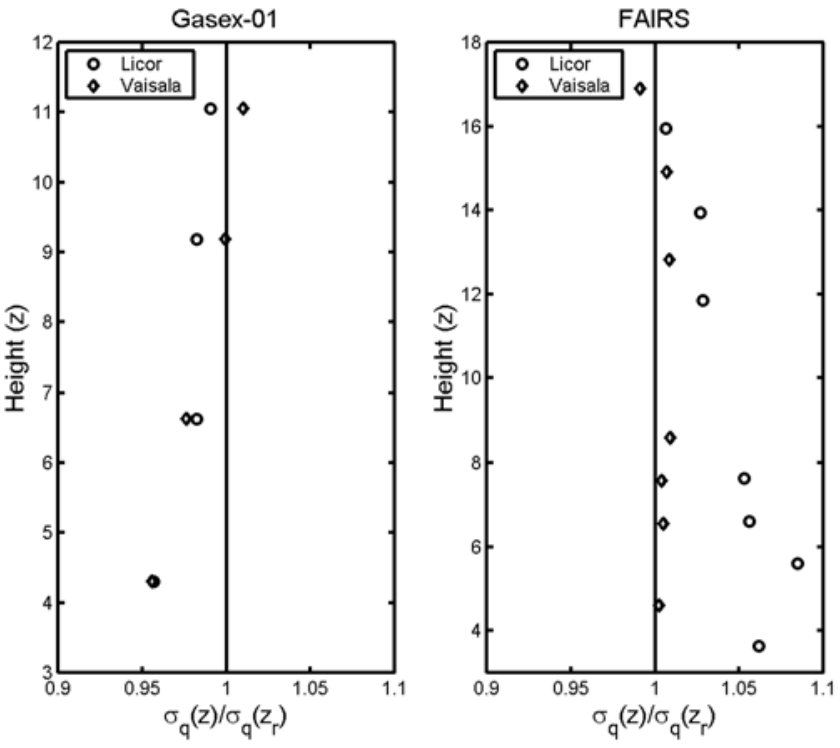

Figure 7. Vertical profiles of the ratio of the standard deviation of the reference to profile values of the specific humidity during the GasEx-01 (left) and FAIRS (right) experiments. Separate profiles for the Licor and Vaisala sensors are shown in each plot.

estimates of $d Q / d \ln (z)$ over the profiling period. Examples of the specific humidity profiles measured in FAIRS and GasEx-01 are shown in Figures 8 and 9.

\subsection{Flow Distortion}

[25] Flow distortion and the need for motion correction leads to larger uncertainties in the fluxes estimated from research platforms. This is particularly true for momentum flux and velocity measurements on research vessels. Scalar flux and profile measurements, however, are expected to be

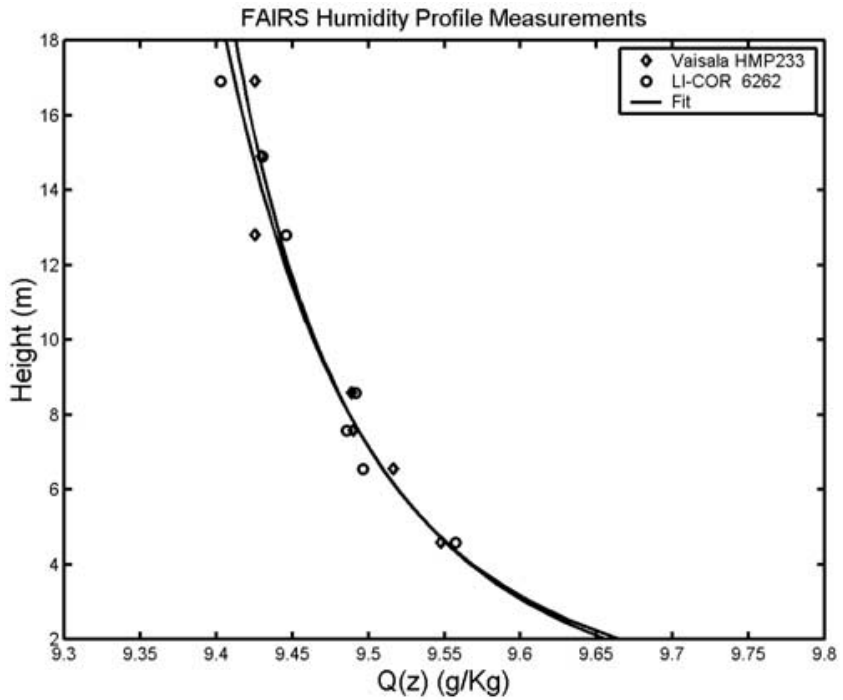

Figure 8. An example of the specific humidity profile measured during the FAIRS experiment for the two humidity sensors used with the profiler. The lines are the second order fits to the data used to compute the gradient. 


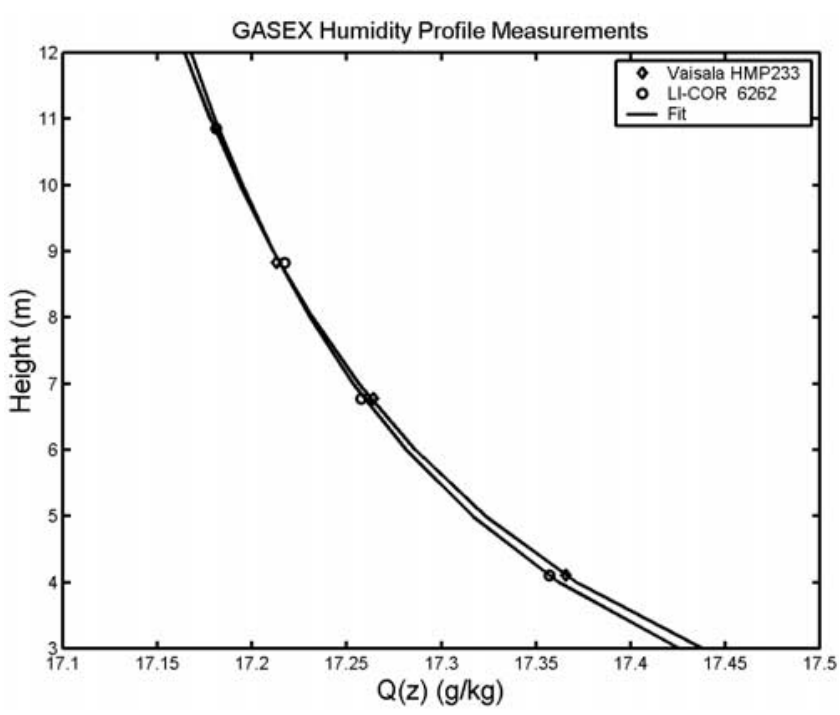

Figure 9. As in Figure 8 for the GasEx experiment.

less sensitive to flow distortion than the velocity measurements [e.g., Wyngaard, 1988; Pedreros et al., 2003]. For this reason this investigation focuses on dimensionless scalar profiles. However, since flow distortion is expected to adversely affect even the scalar flux and profile measurements, this section attempts to quantify the uncertainty in these measurements using results from previous and current investigations.

[26] While the adverse effects of flow distortion around the superstructure and supporting structures are unavoidable on both the R/P FLIP and R/V Brown, this effect can be minimized through careful design of the mounts and placement of the sensors as far away from the superstructure as practical. For scalars, Wyngaard [1988] results indicate that the effect of flow distortion due to the sensor package is negligible if $C_{D}^{1 / 2}(r / \kappa z)^{1 / 3} \ll 1$, where $C_{D}$ is the drag coefficient and $r$ is the size of the sensor package. This condition is easily meet even a few meters above the ocean surface owing to the small size $(r \approx 0.5 \mathrm{~m})$ of the sensor packages and the reduced turbulence intensities (as reflected by $C_{D}^{1 / 2} \approx 0.035$ ) in the marine surface layer.

[27] This criteria does not apply to the flow distortion due to the platform superstructure. Edson et al. [1998] compared fluxes measured on the R/V Wecoma with those computed on the $\mathrm{R} / \mathrm{P}$ FLIP, which requires minimal motion correction and is considered largely distortion-free. Their analysis indicated that the $\mathrm{R} / \mathrm{V}$ Wecoma momentum fluxes were $10-15 \%$ larger than those from the R/P FLIP. After correcting for motion and the systematic increase due to flow distortion, Edson et al. [1998] estimated that the uncertainty ranged from 10 to $20 \%$ for the momentum fluxes and 5 to $10 \%$ for the scalar fluxes (i.e., the uncertainty in $u_{*}$ ). Pedreros et al. [2003] derived similar results from a comparison between the R/V L'Atalante and the R/P $A S I S$ (a largely distortion-free spar buoy). They concluded that the momentum fluxes on the L'Atalante were $18 \%$ higher than ASIS, in good agreement with the Wecoma results. In contrast, the heat fluxes between the L'Atalante and $A S I S$ compared extremely well, indicating that they were less affected by flow distortion.
[28] During GasEx-01, three flux packages were deployed aboard the R/V Brown; the two packages at the end of the bow boom are described above. The third package was deployed by the Environmental Technology Laboratory (ETL) on the forward jackstaff placing the sensors $8.6 \mathrm{~m}$ above the bow and $17.6 \mathrm{~m}$ above the ocean surface. The ETL velocity measurements have been corrected for flow distortion using results from in situ comparisons and computational fluid dynamics (CFD) models [Yelland et al., 2002]. The ratio of the latent heat fluxes, friction velocity, and humidity scaling parameters measured at the two vertical locations as a function of relative wind direction is shown in Figure 10. The median value of these ratios over the relative wind directions used in this analysis is $1.07,1.05$, and 1.00 for the latent heat flux, friction velocity, and humidity scaling velocity, respectively.

[29] Some of the variability in the flux estimates is a result of naturally occurring processes. For example, some of the difference is likely due to the expected decrease of the flux magnitude with height in a typical atmospheric boundary layer. However, the variation in magnitude with relative wind direction is most likely due to flow distortion, and this variability is approximately $10 \%$. Of particular interest for this investigation is the removal of the systematic difference in the humidity scaling parameter after normalization by the friction velocity. This result suggests that any offset due to flow distortion is largely removed from the scalar component after normalization.

[30] Smaller uncertainties in the flux estimates are expected on the R/P FLIP owing to its reduced motion, smaller superstructure, and the ability to place the sensors well away from the superstructure. Edson et al. [1998] found a $9 \%$ difference in the momentum flux measured approximately $3 \mathrm{~m}$ below and $4 \mathrm{~m}$ above the port boom on
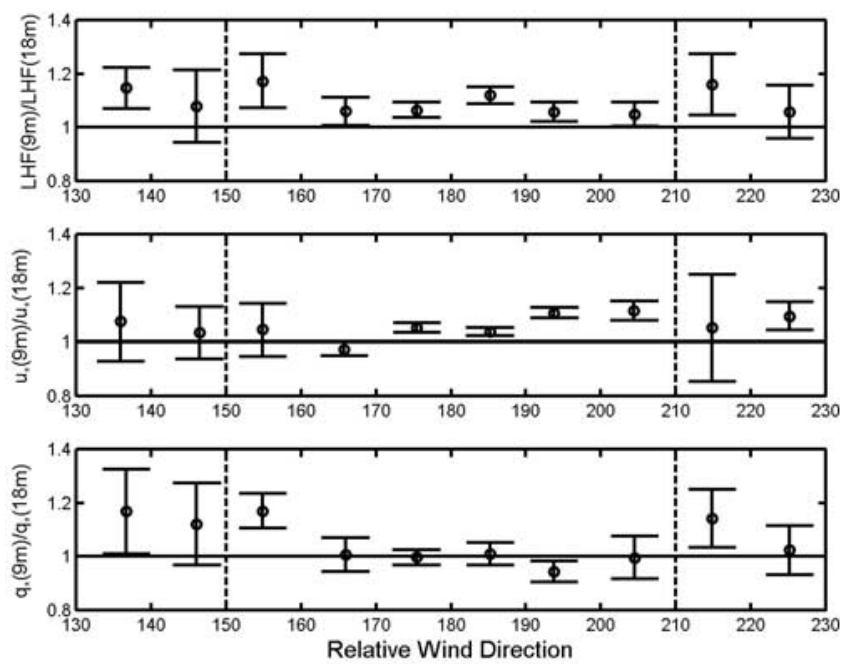

Figure 10. The ratio of the latent heat fluxes (top), friction velocity (middle), and specific humidity scaling parameter (bottom) measured on the bow boom at $9 \mathrm{~m}$ versus the values measured on the jackstaff at $18 \mathrm{~m}$ as a function of the relative wind direction. In this convention, $180^{\circ}$ represents a relative wind direction directly on the bow (i.e., bow to stern). The data shown here represents the range of relative wind directions used in the analysis between $150^{\circ}$ and $210^{\circ}$. 
the $\mathrm{R} / \mathrm{P}$ FLIP. Most of this difference was attributed to naturally occurring variability. Although some fraction of this difference is likely due to flow distortion around the port boom and FLIP itself, these results indicate that the uncertainty in the momentum flux is less than $10 \%$. The uncertainty in the scalar fluxes is expected to be less than $5 \%$.

[31] It is more difficult to quantify the effects of distortion on the mean profiles used in this investigation. Tilting of the flow as it moves over and around the superstructure leads to the possibility that variables measured at given heights originate from different heights in the undisturbed airstream. For example, researchers at the Southampton Oceanography Centre have simulated the flow around a number of research vessels using CFD models [Yelland et al., 2002]. Results from simulations around the $\mathrm{R} / \mathrm{V}$ Brown and the $\mathrm{R} / \mathrm{V}$ Polarstern indicated that the flow is displaced upwards approximately $0.7 \mathrm{~m}$ at the height of the ETL flux package and between 0.4 and 0.15 over the heights of the profiler. This obviously impacts the turbulent properties of the flow and the relationship between the fluxes and profiles. The sensitivity of the dimensionless gradients to the displacement height is investigated in section 4 . In an attempt to further minimize the impact of the flow distortion, the local similarity approach is used in the analysis. Only the local value of the gradient measured at the level of the flux packages is used to compute the dimensionless gradients. This approach compensates for any departure from the constant flux assumption as well as for some of the influence of flow distortion as long as the flux remains proportional to the gradient.

\section{Dimensionless Profiles and Bulk Formulae}

[32] Our direct covariance measurements of sensible heat, latent heat, and momentum fluxes provide estimates of the scaling parameters $u_{*}, q_{*}, T_{*}$, and $L$. Realizing the sensitivity of the velocity profiles to flow distortion, no attempt was made to measure or compute the von Karman's constant. Instead, the conventional value of $\kappa=0.4$ is used in the analysis. This value is slightly larger than the average determined from terrestrial experiments shown in Table 1. However, the larger value is consistent with recent observations of Oncley et al. [1996] and Andreas et al. [2002] that predict even larger values of the von Karman's over the smooth ocean surface (e.g., the range of roughness Reynolds numbers are given by $0.1<R e_{*}<10$ ).

[33] As stated in the introduction, the dimensionless profile function is applicable in any boundary layer flow where the assumptions that govern the MO similarity laws are valid. These assumptions are adequately met in the lowest $10 \%$ of the marine boundary layer above the shallow layer where waves influence the flow, i.e., within the surface layer but above the WBL. The flux measurements used to compute the scaling parameters in these investigations were limited to the height of the flux packages. Although these measurement heights often corresponded to heights where $k_{p} z<1$, the combination of the wave field with the wave heights never provided conditions where the ratio was $z / \sigma_{H}$ smaller than 4 . This is particularly true for the GasEx-01 cruise where the wave field was clearly dominated by nonlocally generated swell. The lowest measurement height in the scalar profiles, however, occasionally extend below the level where $z / \sigma_{H}$ equals 1 . Therefore measurement heights where $z / \sigma_{H}<2$ are excluded from the analysis to insure that the measurements are made above the WBL and to justify the assumptions required for local similarity.

\subsection{Dimensionless Water Vapor Profiles}

[34] The scaling parameters are combined with the measured water vapor profiles to produce estimates of the dimensionless profile function

$$
\phi_{q}(\zeta)=\frac{\kappa z}{q_{*}} \frac{\partial Q}{\partial z}
$$

The analysis only includes estimates for relative wind directions within $\pm 30^{\circ}$ of the bow of the R/V Brown or the keel of the R/V FLIP. Measurements contaminated by rain have also been removed from the analysis. Additionally, analysis of the Vaisala HMP233 data from the GasEx-01 cruise clearly showed that the flow rate provided by the aspirator was not sufficient to overcome the strong solar heating in the equatorial Pacific. Therefore only nighttime values are used in the investigation. The sensitivity of the profiles to the displacement of the streamlines due to flow distortion around the R/V Brown is tested by computing the dimensionless gradients with and without the subtraction of a displacement height given by $\Delta z=0.034 \mathrm{z}$. This parameterization is in good agreement with the CFD results given by Yelland et al. [2002]. Lastly, it is worth noting that the specific humidity measurements are a function of temperature, humidity, and pressure. As with the need to correct temperature profiles for the adiabatic lapse rate, it is important to include the hydrostatic change in pressure to accurately measure the specific humidity profiles. The correction is necessary for the Vaisala measurements, since the sensors move with the profiler. However, it is not necessary for the Licor measurements, since the sensor remains at a fixed height and the sampling tube moves with the profiling package.

[35] Estimates of the dimensionless profiles from the FAIRS and GasEx-01 experiments are shown in Figure 11. Only a few observations were collected under stable conditions during the FAIRS experiment. Therefore it is difficult to shed much light on the neutral value of the turbulent Schmidt number. Instead, the Businger-Dyer and convective formulae are derived by minimizing the mean square error between the data and the functional forms given by equation (7) using $N_{q}=1 / 2$ and $N_{q}=1 / 3$. The fit to the Businger-Dyer scalar formula is done in two ways. In the first approach the y-intercept is assumed to equal 1 (i.e., $\gamma_{q}$ is assumed to equal 1) and the value of $a_{q}$ is varied to minimize the mean square error between the data and corresponding formulation. In the second approach the values of $\gamma_{q}$ and $a_{q}$ are both varied to find the smallest mean square error between the data and formulation. As in the work of Oncley et al. [1996], the stability range $|\zeta|<$ 0.02 is excluded in the analysis to remove the smallest buoyancy fluxes. Additionally, only those observations where $\zeta<-0.1$ are used to determine the free convective form of the equation. This latter stability range includes the lower part of the convective sublayer [Grachev et al., 2000] where the free convection relationship is expected to be valid. 


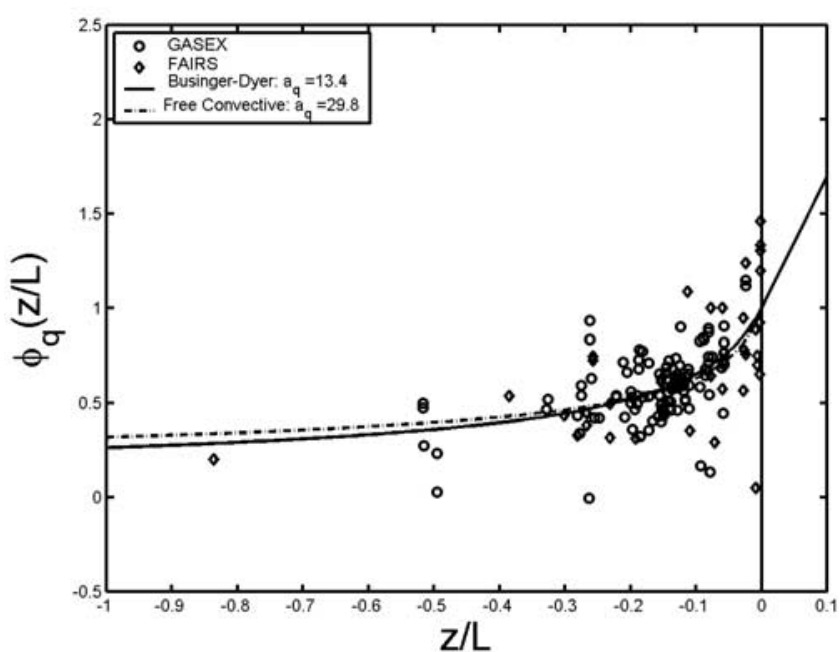

Figure 11. Estimates of the dimensionless water vapor profiles measured during the FAIRS and GasEx experiments plotted against the MO stability parameter. The solid line represents the best fit to the data using the BusingerDyer form of the parameterization, while the broken line represents the best fit to the convective form.

[36] The results from this analysis are summarized in Table 2. The combined data sets give $a_{q}=13.4$ for the Businger-Dyer scalar formula and $a_{q}=28.9$ for the convective form of the parameterization using a value of $\gamma_{q}=1$. When the displacement height is subtracted from the GasEx-01 profiles, the values increase to $a_{q}=13.9$ and 29.9 for the two formula. The six estimates of $a_{q}$ (four from GasEx-01 and two from FAIRS) give a mean and standard deviation of $a_{q}=13.4 \pm 1.7$ and $29.8 \pm 4.6$ for the two formulas. The uncertainty of $12-15 \%$ in these estimates is in good agreement with the uncertainty due to flow distortion and other instrument errors described in section 3.3. These numerical constants also agree with the results from previous overland experiments shown in Table 1. The actual values of $\gamma_{q}$ do not differ significantly from the commonly used value of 1 . The mean and standard deviation of the six values is $\gamma_{q}=1.11 \pm 0.22$. Values that span both unstable and stable conditions are required to reduce the uncertainty in the estimation of $\gamma_{q}$.
[37] The lines in Figure 11 represent the best fits to the data using the two formulae with $\kappa=0.4$ and $\gamma_{q}=1$. The approach outlined by Grachev et al. [2000] is then used to blend these two functions into a single parameterization that applies under all conditions. This function takes the slightly complicated form

$$
\phi_{q}(\zeta)=\frac{\phi_{q B D}(\zeta)+\zeta^{2} \phi_{q C}(\zeta)}{\left(1+\zeta^{2}\right)}+\frac{2 \zeta^{2}\left[\psi_{q B D}(\zeta)-\psi_{q C}(\zeta)\right]}{\left(1+\zeta^{2}\right)^{2}}
$$

where the subscripts $B D$ and $C$ represent the Businger-Dyer and convective forms, respectively, and $\psi_{q}$ is the integral form of the dimensionless gradients given by

$$
\psi_{q}(\zeta)=\int\left[1-\phi_{q}(\zeta)\right] \frac{d \zeta}{\zeta}
$$

This form of $\phi_{q}$ simplifies significantly when integrated to find the form of $\psi_{q}$ used in the profile and bulk aerodynamic methods described below. Equation (14) is plotted against the bin average of the data in Figure 12 using values of $a_{q}=$ 13.4 and 30 in the Businger-Dyer and convective components, respectively. As shown in Figure 12, this formula differs only slightly from the function used in the COARE 3.0 algorithm [Fairall et al., 2003], which uses values of $a_{q}=$ 15 and 34 in the Businger-Dyer and convective components, respectively. These values fall within the uncertainty range of the measurements.

\subsection{Temperature Profiles}

[38] The temperature stratification was near neutral for both the FAIRS and GasEx-01 experiments. The absolute value of the air-sea temperature difference was less than $0.5^{\circ} \mathrm{C}$ for $79 \%$ and $68 \%$ of the time during the FAIRS and GasEx-01 experiments, respectively. As expected, the sensible heat flux estimates are correspondingly small with values averaging $0.5 \mathrm{Wm}^{-2}$ for FAIRS and $5.4 \mathrm{Wm}^{-2}$ for GasEx-01. In fact, the moisture flux component in equation (3) provided more than half of the total buoyancy flux in GasEx-01, and this component kept the surface layer slightly unstable, on average, during FAIRS.

\begin{tabular}{|c|c|c|c|c|c|c|c|c|c|}
\hline \multirow[b]{2}{*}{ Data Set } & \multirow[b]{2}{*}{ Sensor } & \multicolumn{5}{|c|}{$N=1 / 2$} & \multicolumn{3}{|c|}{$N=1 / 3$} \\
\hline & & Number & $\gamma_{q}$ & $a_{q}$ & $\gamma_{q}$ & $a_{q}$ & Number & $\gamma_{q}$ & $a_{q}$ \\
\hline \multirow[t]{3}{*}{ GasEx 2001} & Licor & 69 & 1 & 14.6 & 0.96 & 12.8 & 55 & 1 & 30.1 \\
\hline & Vaisala & 34 & 1 & 11.0 & 1.32 & 25.5 & 25 & 1 & 25.4 \\
\hline & Both & 103 & 1 & 13.3 & 1.14 & 19.6 & 80 & 1 & 28.6 \\
\hline \multirow[t]{3}{*}{ GaseEx $2001 \mathrm{z}-\Delta \mathrm{z}$} & Licor & 69 & 1 & 15.2 & 0.92 & 11.7 & 55 & 1 & 31.2 \\
\hline & Vaisala & 34 & 1 & 11.7 & 1.36 & 29.1 & 25 & 1 & 27.0 \\
\hline & Both & 103 & 1 & 13.9 & 1.12 & 19.5 & 80 & 1 & 29.8 \\
\hline \multirow[t]{3}{*}{ FAIRS 2000} & Licor & 13 & 1 & 14.0 & 1.22 & 26.4 & 7 & 1 & 38.1 \\
\hline & Vaisala & 18 & 1 & 13.7 & 0.88 & 8.5 & 12 & 1 & 26.7 \\
\hline & Both & 31 & 1 & 13.8 & 1.00 & 13.8 & 19 & 1 & 30.5 \\
\hline \multirow[t]{3}{*}{ Combined Data Sets } & Licor & 82 & 1 & 14.5 & 1.08 & 18.3 & 62 & 1 & 30.9 \\
\hline & Vaisala & 52 & 1 & 11.7 & 1.00 & 11.7 & 37 & 1 & 25.8 \\
\hline & Both & 134 & 1 & 13.4 & 1.04 & 15.1 & 99 & 1 & 28.9 \\
\hline \multirow[t]{3}{*}{ Combined Data Sets $z-\Delta z$} & Licor & 82 & 1 & 15.0 & 1.06 & 17.9 & 62 & 1 & 31.9 \\
\hline & Vaisala & 52 & 1 & 12.2 & 1.00 & 12.2 & 37 & 1 & 26.9 \\
\hline & Both & 134 & 1 & 13.9 & 1.04 & 15.7 & 99 & 1 & 29.9 \\
\hline
\end{tabular}

Table 2. Numerical Constants From Regression Analysis Using $\kappa=0.4$ 


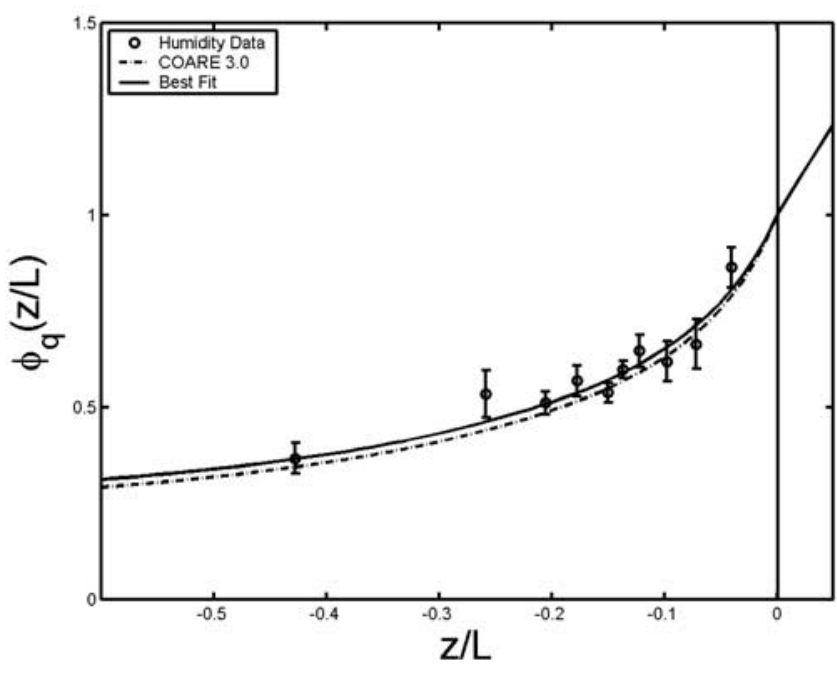

Figure 12. The data shown in Figure 10 bin averaged by stability. Each bin has the same number of data points. The error bars represent the standard error in the measurements. The solid line represents the blended form of the dimensionless shear using the coefficients determined by the best fits. The broken line is the blended form used in the Coupled Ocean-Atmosphere Response Experiment (COARE) 3.0 algorithm.

[39] The small air-sea temperature difference obviously makes it difficult to resolve the temperature profiles, which were often similar in magnitude to the adiabatic lapse rate. This is illustrated in Figure 13, where the top panel represents the dimensionless profile function using the absolute temperature profiles and the bottom panel uses the potential temperature profiles. These plots show that the dimension- less profiles estimates are too large when the uncorrected temperature profiles (i.e., without correction for the adiabatic lapse rate) are used. Nonetheless, even though these small gradients and fluxes generate large uncertainties in these estimates of $\phi_{\theta}(\zeta)$, the cluster of points is centered around the results from the humidity profiles as shown in Figure 14. However, further analysis using this data was not warranted owing to the significant scatter and large experimental uncertainty in these estimates.

\subsection{Bulk Aerodynamic Method}

[40] Perhaps the most common use of the dimensionless profile functions in physical oceanography and marine meteorology is to provide indirect estimates of the fluxes through the bulk aerodynamic, profile, and inertial dissipation methods. The bulk aerodynamic method is undoubtedly the most commonly used approach to estimate the surface fluxes from observations. The method relates the fluxes to the air-sea differences in velocity, humidity, and potential temperature through transfer coefficients as

$$
\begin{gathered}
\tau_{s}=\rho_{a} C_{D} U_{r}^{2} \\
Q_{e}=\rho_{a} L_{e} C_{E}\left[Q\left(z_{o q}\right)-Q(z)\right] U_{r} \\
Q_{h}=\rho_{a} c_{p} C_{H}\left[T_{\left(z_{o \theta}\right)}-\Theta(z)\right] U_{r}
\end{gathered}
$$

where $C_{D}$ is the drag coefficient, $U_{r}$ is the wind speed relative to the ocean surface, and $C_{H}$ and $C_{E}$ are the Stanton and Dalton numbers, respectively. $Q\left(z_{o q}\right)$ and $T\left(z_{o t}\right)$ are the values of the specific humidity and temperature at the surface, respectively, where $z_{o q}$ and $z_{o t}$ are the thermal roughness lengths [e.g., Liu et al., 1979]. The Stanton and
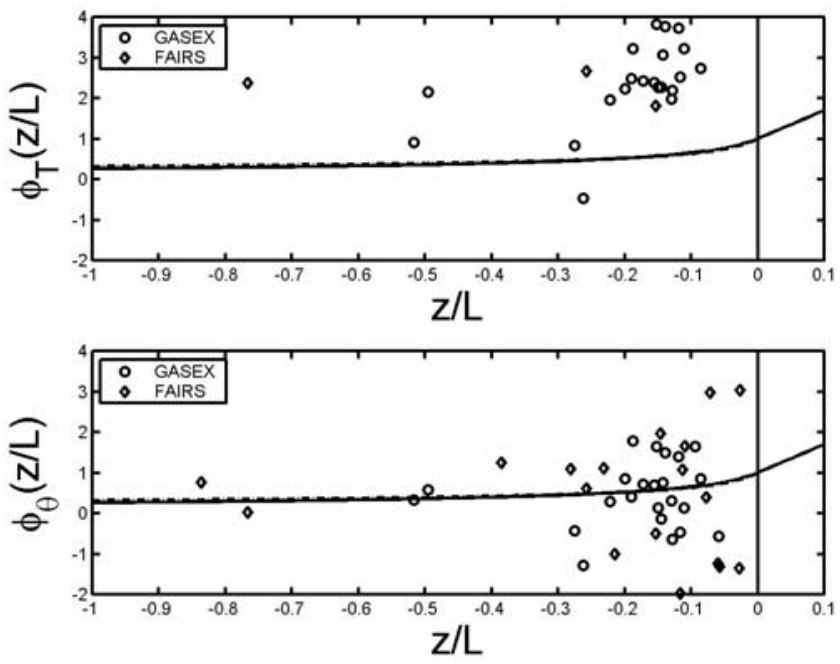

Figure 13. Estimates of the dimensionless temperature (top) and potential temperature (bottom) functions measured during the FAIRS and GasEx experiments plotted against the MO stability parameter. The lines are the same functions shown in Figure 10.

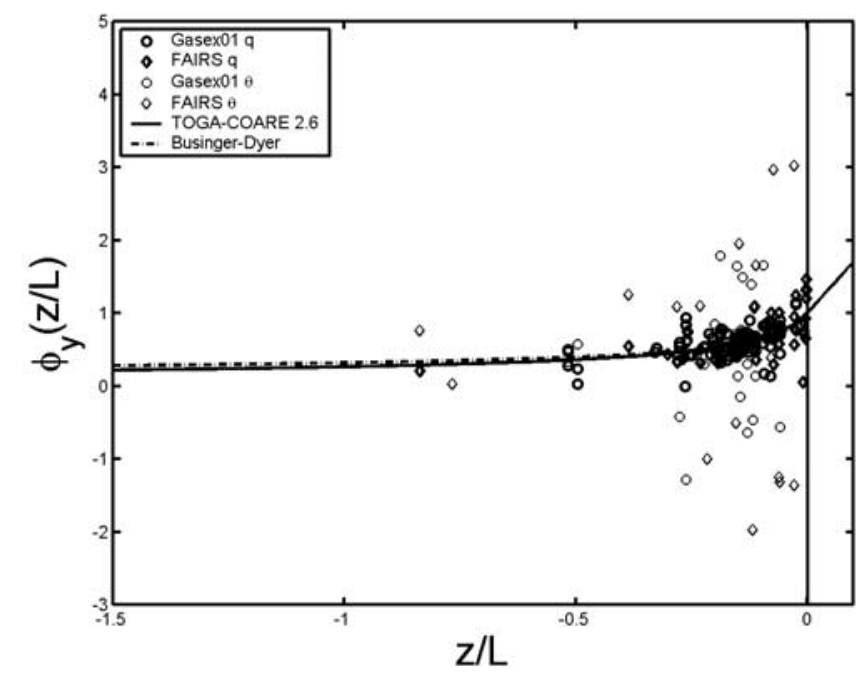

Figure 14. A comparison of the dimensionless water vapor (bold symbols) and potential temperature profiles from the two experiments. The lines are the same functions shown in Figure 10. 


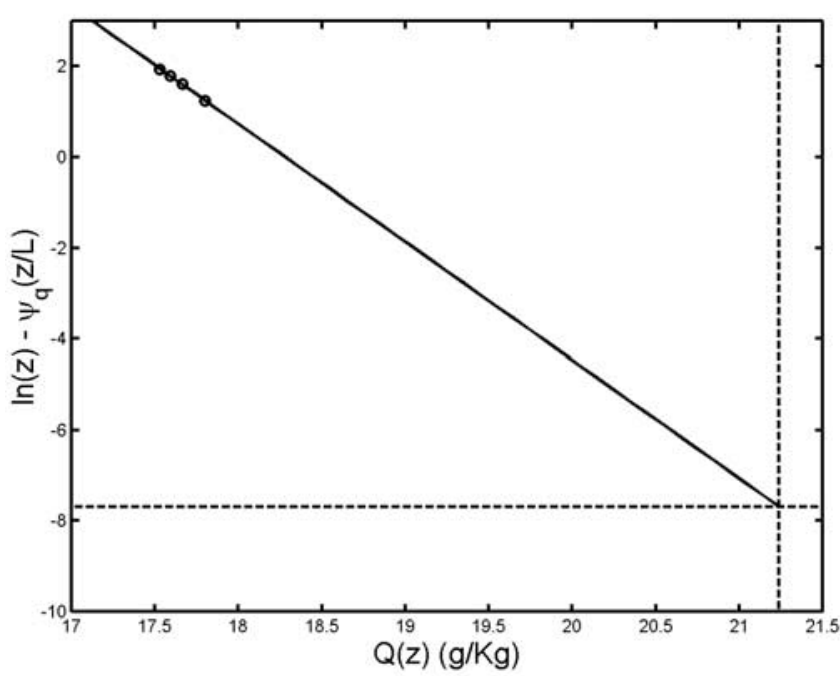

Figure 15. An example of the semilogarithmic fit and extrapolation to the sea surface used to estimate the thermal roughness length. The $\psi_{q}$ function uses the same coefficients found from the best fit analysis.

Dalton numbers can be defined in terms of the drag coefficient and their respective scalar transfer coefficients

$$
\begin{gathered}
C_{H}=C_{D}^{1 / 2} C_{\theta}=C_{u} C_{\theta} \\
C_{E}=C_{D}^{1 / 2} C_{q}=C_{u} C_{q}
\end{gathered}
$$

As stated above, this approach is advantageous because it allows investigators to separate the drag coefficient, which is sensitive to both sea state and wave age, from the scalar transfer coefficients, which are expected to be less influenced by the waves. Instead, these transfer coefficients may be influenced by additional processes such as wave breaking and heat exchange from evaporating sea-spray.

[41] The drag coefficient, Stanton number, and Dalton number are often empirically determined as a function of wind speed [Large and Pond, 1981; Smith, 1988; DeCosmo et al., 1996]. Another common approach is to determine the transfer coefficients using a semiempirical method based on MO similarity theory. The semiempirical forms are derived using the integral form of equation (1) given by

$$
X(z)=X\left(z_{o x}\right)+\frac{x *}{\kappa}\left[\ln \left(\frac{z}{z_{o x}}\right)-\psi_{x}\left(\frac{z}{L}\right)+\psi_{x}\left(\frac{z_{o x}}{L}\right)\right]
$$

where $\psi_{x}\left(z_{o x} / L\right)$ is normally ignored because it is typically much smaller than the other terms. The combination of equation (21) with equations (17) through (20) results in functional forms of the transfer coefficients that depend on stability and the thermal roughness lengths

$$
C_{x}\left(z / z_{o x}, \zeta\right)=\left[\frac{\kappa / \gamma_{x}}{\ln \left(\frac{z}{z_{o x}}\right)-\psi_{x}(\zeta)}\right]
$$

The use of these forms of the transfer coefficient therefore requires parameterizations of $\psi_{x}$ and $z_{o x}$. The integral form of the dimensionless gradient function can be found using the approach given by Fairall et al. [1996]. Integration of equation (14) results in a parameterization with the correct form in the convective limit given by

$$
\psi_{x}(\zeta)=\frac{\psi_{x B D}(\zeta)+\zeta^{2} \psi_{x C}(\zeta)}{1+\zeta^{2}}
$$

where the functions form of $\psi_{x B D}$ and $\psi_{x C}$ are given by Paulson [1970] and Fairall et al. [1996], respectively.

[42] The thermal length scale is commonly parameterized as a function of the roughness Reynold's number [e.g., Liu et al., 1979; Fairall et al., 1996]

$$
z_{\text {oy }}=f\left(\operatorname{Re}_{o}\right)
$$

Field studies have shown that the transfer coefficients for heat and water vapor show substantial scatter and little wind speed dependence [Liu et al., 1979; Smith, 1988]. In fact, the uncertainty in field estimates of the transfer coefficients for temperature and humidity has resulted in the widespread use of laboratory-based parameterizations of $z_{o t}$ for open ocean transfer coefficient models [Liu et al., 1979; Brutsaert, 1982]. However, this is mainly due to the uncertainty in the field measurements rather than an understanding of the physical processes driving the exchange.

[43] Accurate estimates of the sea-surface temperature (SST) or skin temperature, $T\left(z_{o t}\right)$, were computed using infrared techniques [Jessup, 2002] during the FAIRS and GasEx-01 experiments. The SST measurements are used to estimate $Q\left(z_{o q}\right)$ by computing the saturation value for fresh water with a correction factor of 0.97 to account for salinity [Fairall et al., 1996]. This provides an accurate estimate of $Q\left(z_{o q}\right)$ without the need to model the warm layer and cool skin effects required with estimates of the sea temperature measured beneath the surface.

[44] These measurements are then combined with the profile measurements to provide estimates of the thermal roughness length found by extrapolation of the adjusted profiles to the surface values. Specifically, the linear fit of $Q(z)-Q\left(z_{q}\right)$ versus $\ln (z)-\psi_{q}(z / L)$ has a slope of $\kappa / q_{*}$ and a y-intercept of $\ln \left(z_{o q}\right)$. An example of such a fit and extrapolation to the surface value is shown in Figure 15, where the actual values of $Q(z)$ and $Q\left(z_{q}\right)$ are plotted to provide a sense of the air-sea humidity difference during GasEx-01. Values of $z_{o q}$ are plotted versus $R e_{o}$ in Figure 16, where the thermal roughness lengths are computed using the stability function given by equation (23) with the values of $a_{q}$ determined from this investigation. Individual estimates of the roughness lengths are shown in the top panel, which have been estimated with and without subtraction of $\Delta z$. The difference is almost undetectable in the semilogarithmic presentation. The data have been binned into eight ranges where each range has the same number of data points, and the mean, median, and log-average are shown for each bin. The median and log-average values give similar results and show good agreement with the COARE 3.0 parameterization, i.e., the 

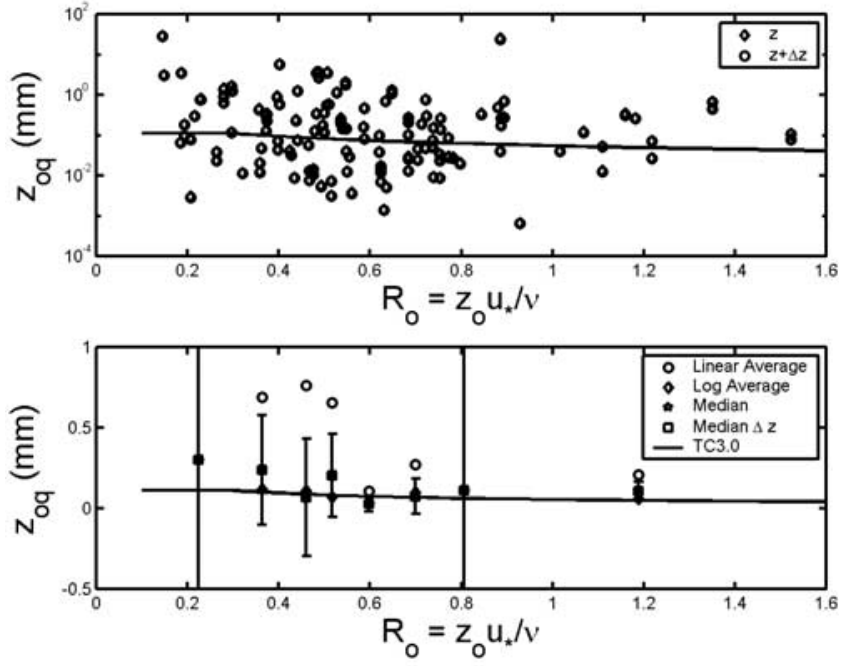

Figure 16. Estimates of the thermal roughness lengths for specific humidity computed from extrapolation to the surface values. Individual estimates of the roughness lengths are shown in the top panel estimated with and without subtraction of $\Delta z$. The bottom panel shows binaveraged estimates where the symbols represent the average, median, and log-average values as indicated and the error bars are the standard error about the median value. The error bars for points 1 and 6 are less than \pm 2 . The solid line is the COARE 3.0 parameterization.

parameterization generally lies within the standard error about these estimates. The plot also shows that the median values with and without the subtraction of $\Delta z$ are very similar. There is some indication that the measurements are slightly larger than predicted by the
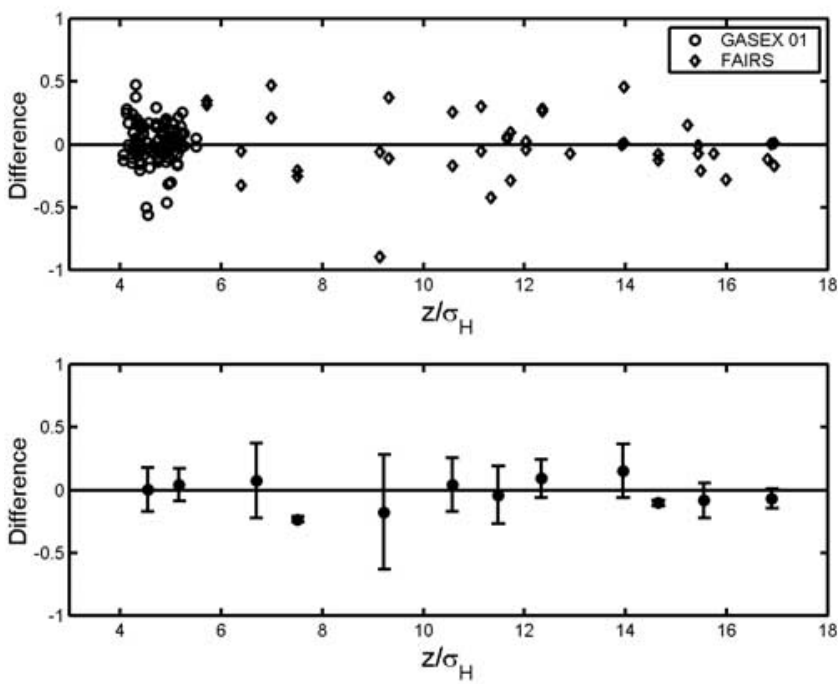

Figure 17. The difference between the individual measurements shown in Figure 10 and the best fit parameterization shown in Figure 11 versus the ratio of the measurement height to significant wave height $z / \sigma_{H}$. parameterization. However, the large uncertainties in these estimates require additional measurements to modify this parameterization.

\subsection{Sea State and the WBL for Scalars}

[45] The setups used in FAIRS and GasEx-01 were not designed to investigate the role of waves on the scalar flux-profile relationships. However, the results can be used to provide evidence that the measurements were made above the WBL for heat and mass transfer. For example, the difference between the individual measurements of $\kappa z / q_{*} \partial Q / \partial z$ and the best-fit parameterization of $\phi_{q}(\zeta)$ given by equation (14) can be plotted against several commonly used sea-state parameters to investigate whether the residual is correlated with these parameters. The residual is plotted against $z / \sigma_{H}$ and $k_{p} z$ in Figures 17 and 18. The individual points and bin-averaged data in the upper and lower panel, respectively, show little or no dependence on these wave-related parameters in the swell-dominated wave field often encountered over the open ocean. These results support the conclusion that the measurements are made above the WBL for heat and mass exchange.

[46] These results indicate that the turbulence responsible for transporting heat and mass is locally generated by shear and buoyancy. This is a necessary requirement for application of MO similarity. However, these results do not necessary imply that the scalar fluxes are uninfluenced by waves at all heights. For example, it is well known that the momentum flux and $u_{*}$ are expected to be a function of sea state. However, normalization of the fluxes by is expected to significantly reduce the effect of waves on the scalar component of the flux, i.e., $q_{*}$ and $T_{*}$. Investigations of the influence of waves on the scalar fluxes and flux profile relationship therefore require a
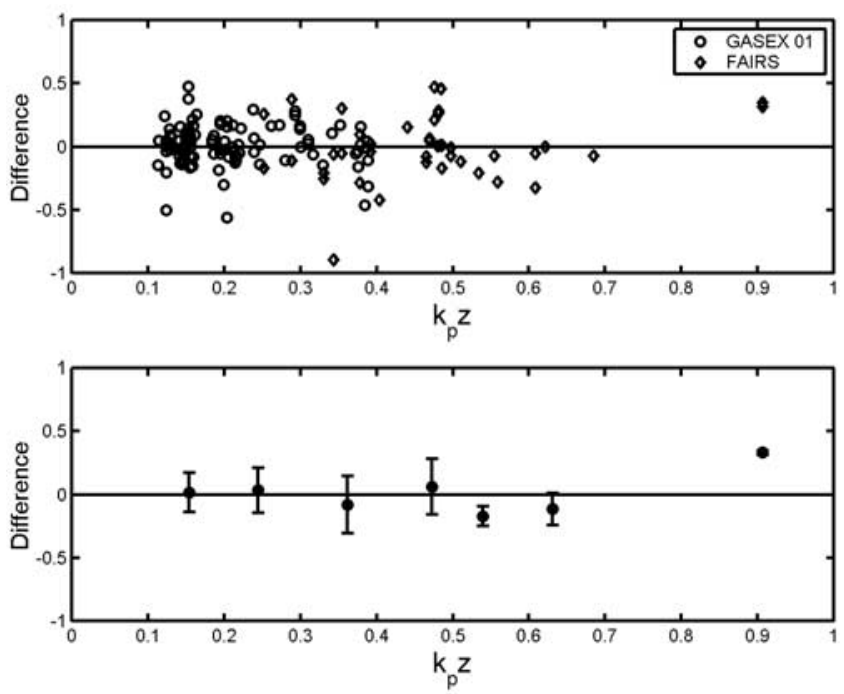

Figure 18. The difference between the individual measurements shown in Figure 10 and the best fit parameterization shown in Figure 11 versus the dimensionless height, $k_{p} z$, which equals the ratio of the measurement height to wave length times $2 \pi$. 
setup with profiles and fluxes measured much closer to the air-sea interface.

\section{Conclusions}

[47] Our open ocean estimates are in good agreement with commonly used parameterizations based on overland measurements. This indicates that the MO similarity functions are applicable over the ocean where appropriate, i.e., in the surface layer above the WBL where the structure of the turbulence is dominated by the relative importance of mechanical (i.e., wind shear) versus thermal forcing. In the analysis a value of $a_{q}=13.4$ gives good agreement with the data using Businger-Dyer forms of the equations in near-neutral conditions. Using the approach outlined by Fairall et al. [1996] and Grachev et al. [2000], the blended parameterization shows good agreement with the data, using a value of $a_{q}=30$ for the convective component of the dimensionless gradient. The resulting functions are not significantly different from the latest parameterizations of the dimensionless gradients used in the COARE 3.0 algorithm [Fairall et al., 2003], and the results are in good agreement with the consensus values found from previous overland experiments shown in Table 1.

[48] Although the flux measurements were made at a single elevation and local similarity scaling is applied, the good agreement implies that MO similarity is valid within the marine atmospheric surface layer. The results also indicate that the scalar fluxes are not influenced by wave-induced effects at the measurement heights, i.e., the measurements were taken above the WBL for scalars. As a result, the dimensionless gradients based on MO similarity can be used to compute scalar fluxes in the region of the surface layer above the scalar WBL. This region corresponds to typical measurement heights aboard research vessels. To make this same claim for buoys and other platforms that place the sensors closer to the surface would obviously require profile and flux measurements closer to the surface.

[49] The thermal roughness lengths determined from extrapolation of the semilogarithmic profiles to the surface values are also in good agreement with the COARE 3.0 algorithm. There is some indication that the COARE 3.0 algorithm overestimates the values of the thermal roughness lengths at low Reynold's numbers. However, the large uncertainty in this extrapolation caused by the accumulated effect of the various experimental errors makes it difficult to quantify this observation. The reduction of this uncertainty is an objective of the recently conducted Coupled Boundary Layers and Air-Sea Transfer (CBLAST) experiment at the Martha's Vineyard Coastal Observatory.

[50] Acknowledgments. The FAIRS work was supported by the Office of Naval Research grant N00014-00-1-0403 while the GasEx work was supported by the National Science Foundation grant OCE-9986724. The authors wish to recognize the crews of the R/P FLIP and NOAA Ship Ronald H. Brown for their outstanding efforts. We would like to thank Steve Faluotico, Sean McKenna, and Glenn McDonald from WHOI for their help in developing and maintaining the flux-profile system during the FAIRS and GasEx cruises. We would like to thank Andrew Jessup from UW/APL and Brian Ward from NOAA/AOML for their assistance with the sea surface and bulk temperature measurements. Finally, we would like to thank Sheila Hurst for her editorial assistance. This is Woods Hole Oceanographic Institution contribution 10925.

\section{References}

Andreas, E. L., and K. A. Emanuel (2001), Effects of sea spray on tropical cyclone intensity, J. Atmos. Sci., 58, 3741-3751.

Andreas, E. L., K. J. Claffey, C. W. Fairall, P. S. Guest, R. E. Jordan, and P. O. G. Persson (2002), Evidence from the atmospheric surface layer that the von Karman constant isn't, in Fifteenth Symposium on Boundary Layers and Turbulence, pp. 418-421, Am. Meteorol. Soc., Boston.

Badgley, F. I., and C. A. Paulson (1972), Profiles of wind, temperature and humidity over the Arabian Sea I: The observations, Int. Indian Ocean Exped. Meteor. Mongr., 6, 3-29.

Brutsaert, W. (1982), Evaporation into the Atmosphere, 299 pp., D. Reidel, Norwell, Mass.

Businger, J. A. (1988), A note on the Businger-Dyer profiles, Boundary Layer Meteorol., 42, 145-151.

Businger, J. A., J. C. Wyngaard, Y. Izumi, and E. F. Bradley (1971), Fluxprofile relations in the atmospheric surface layer, J. Atmos. Sci., 28, $181-$ 189

Carl, M. D., T. C. Tarbell, and H. A. Panofsky (1973), Profiles of wind and temperature from towers over homogeneous terrain, J. Atmos. Sci., 30, $788-794$.

DeCosmo, J., K. B. Katsaros, S. D. Smith, R. J. Anderson, W. A. Oost, K. Bumke, and H. Chadwick (1996), Air-sea exchange of water vapor and sensible heat: The Humidity Exchange over the Sea (HEXOS) results, J. Geophys. Res., 101, 12,001-12,016.

Dyer, A. J., and E. F. Bradley (1982), An alternative analysis of fluxgradient relationships at the 1976 ITCE, Boundary Layer Meteorol., $22,3-19$.

Dyer, A. J., and B. B. Hicks (1970), Flux-gradient relationships in the constant flux layer, Q. J. R. Meteorol. Soc., 96, 715-721.

Edson, J. B., and C. W. Fairall (1998), Similarity relationships in the marine surface layer, J. Atmos. Sci., 55, 2311-2328.

Edson, J. B., A. A. Hinton, K. E. Prada, J. E. Hare, and C. W. Fairall (1998), Direct covariance flux estimates from mobile platforms at sea, J. Atmos. Ocean. Technol., 15, 547-562.

Fairall, C. W., J. D. Kepert, and G. J. Holland (1994), The effect of sea spray on surface energy transports over the ocean, Global Atmos. Ocean Sys., 2, 121-142.

Fairall, C. W., E. F. Bradley, D. P. Rogers, J. B. Edson, and G. S. Young (1996), Bulk parameterization of air-sea fluxes for TOGA COARE, J. Geophys. Res., 101, 3747-3764.

Fairall, C. W., E. F. Bradley, J. E. Hare, A. A. Grachev, and J. B. Edson (2003), Bulk parameterization of air-sea fluxes: Updates and verification for the COARE algorithm, J. Clim., 16, 571-591.

Frenzen, P., and C. A. Vogel (1992), The turbulent kinetic energy budget in the atmospheric surface layer: A review and an experimental reexamination in the field, Boundary Layer Meteorol., 60, 49-76.

Frenzen, P., and C. A. Vogel (2001), Further studies of atmospheric turbulence in layers near the surface: Scaling the TKE budget above the roughness sublayer, Boundary Layer Meteorol., 99, 173-206.

Grachev, A. A., C. W. Fairall, and E. F. Bradley (2000), Convective profile constants revisited, Boundary Layer Meteorol., 94, 495-515.

Gurvich, A. S. (1965), Vertical temperature and wind velocity profiles in the atmospheric surface layer, Izv. Acad. Sci. USSR Atmos. Oceanic Phys., Engl. Transl., 1, 31-36.

Hare, J. E., T. Hara, J. B. Edson, and J. M. Wilczak (1997), A similarity analysis of the structure of air flow over surface waves, J. Phys. Oceanogr., 27, 1018-1037.

Högström, U. (1988), Non-dimensional wind and temperature profiles in the atmospheric surface layer: A re-evaluation, Boundary Layer Meteorol., 42, 55-78.

Hristov, T. S., S. D. Miller, and C. A. Friehe (2003), Dynamical coupling of wind and ocean waves through wave-induced air flow, Nature, 422, $55-$ 58 .

Janssen, P. A. E. M. (1989), Wave induced stress and the drag of the air flow over sea waves, J. Phys. Oceanogr., 19, 745-754.

Janssen, P. A. E. M. (1999), On the effect of ocean waves on the kinetic energy balance and consequences for the inertial dissipation technique, J. Phys. Oceanogr., 29, 530-534.

Jessup, A. T. (2002), Autonomous shipboard infrared radiometer system for in situ validation of satellite SST, Proc. SPIE, 4814, 222-229.

Large, W. G., and S. Pond (1981), Open ocean momentum flux measurements in moderate to strong winds, J. Phys. Oceanogr., 11, 324336.

Liu, W. T., K. B. Katsaros, and J. A. Businger (1979), Bulk parameterization of air-sea exchanges of heat and water vapor including the molecular constraints at the interface, J. Atmos. Sci., 36, 1722-1735.

Mahrt, L. (1998), Stratified atmospheric boundary layers, Boundary Layer Meteorol., 90, 375-396.

Makin, V. K. (1998), Air-sea exchange of heat in the presence of wind waves and spray, J. Geophys. Res., 103, 1137-1152. 
Makin, V. K., and C. Mastenbroek (1996), Impact of waves on air-sea exchange of sensible heat and momentum, Boundary Layer Meteorol. 79, 279-300

Makin, V. K., V. N. Kudryavtsev, and C. Mastenbroek (1995), Drag of the sea surface, Boundary Layer Meteorol., 73, 159-182.

Miller, S. D., C. A. Friehe, T. S. Hristov, and J. B. Edson (1997), Wind and turbulence profiles in the surface layer over the ocean, in Twelfth Symposium on Boundary Layers and Turbulence, pp. 308-309, Am. Meteorol. Soc., Boston.

Miyake, M., M. Donelan, G. McBean, C. Paulson, F. Badgley, and E. Leavitt (1970), Comparison of turbulent fluxes over water determined by profile and eddy correlation techniques, Q. J. R. Meteorol. Soc., 96, $132-137$

Oncley, S. P., C. A. Friehe, J. C. LaRue, J. A. Businger, E. C. Itsweire, and S. S. Cheng (1996), Surface-layer fluxes, profiles, and turbulence measurements over uniform terrain under near-neutral conditions, J. Atmos. Sci., 53, 1029-1044.

Panofsky, H. A., A. K. Blackadar, and G. E. McVehil (1960), The diabatic wind profile, O. J. R. Meteorol. Soc., 86, 495-503.

Paulson, C. A. (1970), Mathematical representation of wind speed and temperature profiles in the unstable atmospheric surface layer, $J . A p p l$. Meteorol., 9, 857-861.

Paulson, C. A., M. Miyake, and F. I. Badgley (1972a), Profiles of wind, temperature and humidity over the Arabian Sea II: An analysis, Intern. Indian Ocean Exped. Meteor. Mongr., 6, 33-62.

Paulson, C. A., E. Leavitt, and R. G. Fleagle (1972b), Air-sea transfer of momentum, heat and water determined from profile measurements during BOMEX, J. Phys. Oceanogr., 2, 487-497.

Pedreros, R., G. Dardier, H. Dupuis, H. C. Graber, W. M. Drennan, A. Weill, C. Guérin, and P. Nacass (2003), Momentum and heat fluxes via the eddy correlation method on the R/V L'Atalante and an ASIS buoy, J. Geophys. Res., 108(C11), 3339, doi:10.1029/2002JC001449.

Pond, S., G. T. Phelps, J. E. Paquin, G. McBean, and R. W. Stewart (1971) Measurements of the turbulent fluxes of momentum, moisture and sensible heat over the ocean, J. Atmos. Sci., 28, 901-917.

Poulos, G. S., et al. (2002), CASES-99: A comprehensive investigation of the stable nocturnal boundary layer, Bull. Am. Meteorol. Soc., 83, $555-$ 581
Smedman, A., U. Högström, H. Bergström, A. Rutgersson, K. K. Kahma, and H. Pettersson (1999), A case study of air-sea interaction during swell conditions, J. Geophys. Res., 104, 25,833-25,851.

Smith, S. D. (1988), Coefficients for sea surface wind stress, heat flux, and wind profiles as a function of wind speed and temperature, J. Geophys. Res., 93, 15,467-15,472.

Stull, R. B. (1988), An Introduction to Boundary Layer Meteorology, 666 pp., Kluwer Acad., Norwell, Mass.

Vickers, D., and L. Mahrt (1999), Observations of non-dimensional wind shear in the coastal zone, O. J. R. Meteorol. Soc., 125, 2685-2702.

Wyngaard, J. C. (1973), On surface layer turbulence, in Workshop on Micrometeorology, edited by D. A. Haugen, pp. 101-149, Am. Meteorol. Soc., Boston.

Wyngaard, J. C. (1988), The effects of probe-induced flow distortion on atmospheric turbulence measurements: Extension to scalars, J. Atmos. Sci., 45, 3400-3412.

Yaglom, A. M. (1977), Comments on wind and temperature flux-profiles relationships, Boundary Layer Meteorol., 11, 89-102.

Yelland, M. J., B. I. Moat, R. W. Pascal, and D. I. Berry (2002), CFD model estimates of the airflow distortion over research ships and the impact on momentum flux measurements, J. Atmos. Oceanic Technol., 19, $1477-$ 1499.

J. B. Edson and J. A. Ware, Woods Hole Oceanographic Institution, 98 Water Street, Woods Hole, MA 02543, USA. (jedson@whoi.edu; jware@whoi.edu)

J. E. Hare, Environmental Technologies Laboratory, National Oceanic and Atmospheric Administration, 325 Broadway R/ETL, Boulder, CO 80305-3328, USA. (jeff.hare@noaa.gov)

W. R. McGillis, Geochemistry Division, Lamont-Doherty Earth Observatory, Columbia University, 208 Geoscience, 61 Route 9W, P. O. Box 1000, Palisades, NY 10964-8000, USA. (mcgillis@1deo.columbia. edu)

C. J. Zappa, Ocean and Climate Physics Division, Lamont-Doherty Earth Observatory, Columbia University, 204D Oceanography, 61 Route 9W, P. O. Box 1000, Palisades, NY 10964-8000, USA. (zappa@ldeo.columbia.edu) 